\title{
Tilings of Polygons with Similar Triangles, II*
}

\author{
M. Laczkovich \\ Department of Analysis, Eötvös Loránd University, \\ Muzeum krt. 6-8, 1088 Budapest, Hungary \\ laczk@cs.elte.hu
}

Communicated by János Pach

\begin{abstract}
Let $A$ be a polygon, and let $s(A)$ denote the number of distinct nonsimilar triangles $\Delta$ such that $A$ can be dissected into finitely many triangles similar to $\Delta$. If $A$ can be decomposed into finitely many similar symmetric trapezoids, then $s(A)=\infty$. This implies that if $A$ is a regular polygon, then $s(A)=\infty$. In the other direction, we show that if $s(A)=\infty$, then $A$ can be decomposed into finitely many symmetric trapezoids with the same angles.

We introduce the following classification of tilings: a tiling is regular if $\Delta$ has two angles, $\alpha$ and $\beta$, such that at each vertex of the tiling the number of angles $\alpha$ is the same as that of $\beta$. Otherwise the tiling is irregular. We prove that for every polygon $A$ the number of triangles that tile $A$ irregularly is at most $c \cdot n^{6}$, where $n$ is the number of vertices of $A$. If $A$ has a regular tiling, then $A$ can be decomposed into finitely many symmetric trapezoids with the same angles.
\end{abstract}

\section{Introduction}

We say that a triangle $\Delta$ tiles the polygon $A$, if $A$ can be decomposed into finitely many nonoverlapping triangles similar to $\Delta$. The investigations of this paper were motivated by the following general problem: Given a polygon $A$, determine the set $S(A)$ of triangles that tile $A$.

The first paper that was devoted to this problem seems to be [2] (see also [7, Chap. 6]), although some related questions were considered earlier. Soifer's book [6] contains several results concerning tilings of triangles. He proves, e.g., that every triangle $T$ can

\footnotetext{
* This research was supported by the Hungarian National Foundation for Scientific Research, Grant T019476.
} 
be cut into any number $n$ of similar triangles, except $n=2,3,5$. In [1], Golomb conjectured that if the pieces must be congruent to each other and similar to the triangle $T$, then the number of pieces is necessarily $n^{2}, 3 n^{2}$, or $n^{2}+k^{2}$. This was proved by Snover et al. in [5].

In [3] we proved that if $T$ is a nonequilateral triangle, then $S(T)$ consists of at most six triangles, and if $T$ is the equilateral triangle, then $S(T)$ is countably infinite. The exact determination of $S(A)$ for a general polygon $A$ is difficult. In fact, this problem is unsolved even in the simplest cases, e.g., when $A$ is a square. However, in [2] we proved that if a triangle $\Delta$ tiles the square then, apart from five exceptional cases, $\Delta$ must be a right triangle such that the acute angles of $\Delta$ are not rational multiples of $\pi$ (see Theorem 23 and Section 6 of [2]). Let $\Delta$ be such a right triangle with acute angles $\alpha$ and $\beta$. Then every tiling of the square with similar copies of $\Delta$ is necessarily regular in the following sense:

For every vertex $V$ of the tiling, the number of those triangles of the tiling that have angle $\alpha$ at $V$ must be the same as the number of triangles having angle $\beta$ at $V$.

Indeed, summing up the angles meeting at $V$ we obtain an equation of the form $p \alpha+q \beta+r(\pi / 2)=v \pi$, where $p, q, r$ are nonnegative integers, $v=1 / 2$ if $V$ is a vertex of the square, and $v \in\{1,2\}$ otherwise. If $p \neq q$, then this would imply that $\alpha$ is a rational multiple of $\pi$, contrary to the hypothesis.

In this paper we shall prove that this behavior of the square is typical. That is, for every polygon $A$, the number of those triangles $\Delta \in S(A)$ for which there is an irregular tiling of $A$ with similar copies of $\Delta$ is finite; more exactly, it is bounded by a function of the number of vertices of $A$ (Theorem 4 ). The proof of Theorem 4 gives an effective finite set containing every triangle $\Delta$ such that $A$ has an irregular tiling with similar copies of $\Delta$. This makes it possible - at least in principle — to determine the exact set of these triangles.

In spite of the seemingly simple structure of regular tilings, the problem of listing those triangles that tile a polygon regularly is more difficult. (This problem is unsolved even for the square; see [2] and [4].) However, we shall prove that the mere existence of a regular tiling imposes a strong condition on the polygon $A$ : in this case there must exist a tiling of $A$ with symmetric trapezoids of the same angles (Theorem 3 ).

Let $s(A)$ denote the cardinality of $S(A)$. In [2, Cor. 15] we proved that $s(A) \leq \aleph_{0}$ for every polygon. If $s(A)=\aleph_{0}$, then there are regular tilings of $A$ and, consequently, $A$ can be tiled with symmetric trapezoids of the same angles (Corollary 5 ). In the other direction, the following proposition gives a simple sufficient condition for $s(A)=\aleph_{0}$. In the sequel we shall write $s(A)=\infty$ instead of $s(A)=\aleph_{0}$.

Proposition 1. If a polygon A can be decomposed into finitely many similar symmetric trapezoids, then $s(A)=\infty$.

Proof. It is enough to show that if $T$ is a symmetric trapezoid then $s(T)=\infty$. Let the vertices of a symmetric trapezoid $T$ be $A, B, C, D$, listed counterclockwise, and such that $A B$ and $C D$ are the bases of $T$.

First we prove that if $\overline{D C} \geq 2 \cdot \overline{A D}$, then $T$ can be decomposed into three similar triangles. Let $\angle A D C=\gamma$. If $M$ is the middle point of the segment $D C$, then $\angle A M B \geq$ 
$\gamma$. Indeed, $\overline{D M} \geq \overline{D A}$ implies $\angle D A M \geq \angle D M A=\angle M A B$, and thus

$$
\angle A M B=\pi-2 \cdot \angle M A B \geq \pi-\angle D A M-\angle D M A=\gamma,
$$

as we stated. On the other hand, $\angle A D B<\gamma$, and thus there is a point $P$ on the segment $D M$ such that $\angle A P B=\gamma$. Then $\angle D P A=\angle P A B$ and $\angle A B P=\angle B P C$, so that the triangles $D A P, A B P, P B C$ are similar to each other.

Now let $T=A B C D$ be an arbitrary symmetric trapezoid with bases $A B$ and $C D$. Then for every $n$ there are points $P_{0}=A, P_{1}, \ldots, P_{n}=D$, and $Q_{0}=B, Q_{1}, \ldots, Q_{n}=$ $C$ on the segments $A D$ and $B C$, respectively, such that the trapeziods $T_{i}=P_{i} Q_{i} Q_{i+1} P_{i+1}$ are similar to each other. If $n$ is large enough, then $\overline{P_{i} Q_{i}} \geq 2 \cdot \overline{P_{i} P_{i+1}}$ and thus $T_{i}$ can be decomposed into three similar triangles. That is, $T$ can be decomposed into $3 n$ similar triangles for every $n \geq n_{0}$. Since the smallest angle of these triangles tends to zero if $n \rightarrow \infty$, we obtain $s(T)=\infty$.

As an application of Proposition 1, we prove the following result:

Theorem 2. Let $R_{n}$ denote the regular $n$-gon. Then $s\left(R_{n}\right)=\infty$ for every $n \geq 3$.

Proof. It is enough to show that $R_{n}$ can be decomposed into similar symmetric trapezoids. First we note that if $3 \leq n \leq 6$, then $R_{n}$ can be decomposed into congruent symmetric trapezoids. This is clear for $n=4$, as $R_{4}$ is a symmetric trapezoid itself. The case of $n=6$ is also simple, since $R_{6}$ is decomposed into two congruent symmetric trapezoids by joining two opposite vertices. As for $n=3$, let $P_{0}, P_{1}, P_{2}, P_{3}=P_{0}$ be the vertices of an equilateral triangle $T$ and let $O$ be the center of $T$. Let $Q_{i}$ be a point on the segment $P_{i-1} P_{i}$ such that $O Q_{i}$ is parallel to $P_{i} P_{i+1} \quad(i=1,2,3)$. Then the segments $O Q_{i}(i=1,2,3)$ decompose $T$ into three congruent symmetric trapezoids.

The construction for a regular pentagon $P_{0}, \ldots, P_{5}=P_{0}$ is similar. Let $O$ be the center of the pentagon, and let $Q_{i}$ be a point on the segment $P_{i-1} P_{i}$ such that $O Q_{i}$ is parallel to $P_{i} P_{i+1}(i=1, \ldots, 5)$. Then the segments $O Q_{i}(i=1, \ldots, 5)$ decompose the regular pentagon into five congruent symmetric trapezoids.

This construction does not work for $R_{n}$ if $n>6$, and it can be shown that for $n \geq 7$ (with the possible exception of $n=8$ and 12) the regular polygon $R_{n}$ cannot be decomposed into congruent symmetric trapezoids. In order to decompose $R_{n}$ into similar symmetric trapezoids, we need a different construction. We shall give the details only for $n=7$.

Let $O$ denote the origin of the coordinate system in the plane, and let $\alpha=2 \pi / 7$. For every positive real number $x$, the points $O, P_{1}(x+2 \cos \alpha, 0), P_{2}(x+\cos \alpha, \sin \alpha)$, $P_{3}(\cos \alpha, \sin \alpha)$ constitute the vertices of a symmetric trapezoid $T_{1}(x)$. There are points $P_{5}$ and $P_{6}$ such that the points $P_{4}(1,0), P_{5}, P_{6}, P_{1}$ constitute the vertices of a symmetric trapezoid $T_{2}(x)$ listed counterclockwise and such that $T_{1}(x)$ and $T_{2}(x)$ are similar and nonoverlapping. Then $P_{2}, P_{1}, P_{6}$ are collinear. Thus there are points $P_{7}$ and $P_{8}$ such that $P_{2}, P_{6}, P_{7}, P_{8}$ constitute the vertices of a symmetric trapezoid $T_{3}(x)$ similar to $T_{1}(x)$. Then $P_{3}, P_{2}, P_{8}$ are collinear. Let $\overline{P_{3} P_{8}}=y(x)$ and $\overline{P_{4} P_{5}}=z(x)$. Clearly, $y$ and $z$ are 
continuous functions of $x$. A simple computation shows that

$$
\lim _{x \rightarrow+0} y(x)=\frac{1}{2 \cos \alpha}>4 \cos ^{2} \alpha-2 \cos \alpha=\lim _{x \rightarrow+0} z(x) .
$$

On the other hand, $z(x)>y(x)$ if $x$ is large enough. Indeed, the $x$-coordinate of $P_{5}$ is greater than that of $P_{8}$, and thus

$$
z(x)>\frac{y(x)-1}{\cos \alpha}>y(x)
$$

if $y(x)>1 /(1-\cos \alpha)$. This implies that $y(x)=z(x)$ for a suitable positive $x$. Fixing such an $x$, the hexagon $H=0 P_{4} P_{5} P_{7} P_{8} P_{3}$ is the union of three similar symmetric trapezoids, and has the property that $\overline{O P_{4}}=\overline{O P_{3}}=1$, and $\overline{P_{4} P_{5}}=\overline{P_{3} P_{8}}$. Since the angle of $H$ at the vertex $P_{7}$ is $5 \pi / 7$, this easily implies that if we rotate $H$ about the origin $O$ with angles $2 \pi i / 7(i=0,1, \ldots, 6)$, then the hexagons obtained in this way constitute a partition of a regular 7-gon. This proves that $R_{7}$ can be decomposed into 21 similar symmetric trapezoids. The same construction shows (using $\alpha=2 \pi / 8$ instead of $2 \pi / 7)$ that $R_{8}$ can be decomposed into 24 similar symmetric trapeziods. For $n \geq 9$ we need a more complicated construction involving a longer chain of similar symmetric trapeziods. We omit the details.

\section{Regular and Irregular Tilings}

Let $T$ be a decomposition of a polygon $A$ into nonoverlapping similar triangles $\Delta_{1}, \ldots, \Delta_{N}$ of angles $\alpha, \beta, \gamma$. Let $V_{1}, \ldots, V_{m}$ be an enumeration of the vertices of the triangles $\Delta_{1}, \ldots, \Delta_{N}$. For every $i=1, \ldots, m$ we shall denote by $p_{i}$ (resp., $q_{i}$ or $r_{i}$ ) the number of those triangles $\Delta_{j}$ whose angle at the vertex $V_{i}$ is $\alpha$ (resp., $\beta$ or $\gamma$ ). If $i \leq n$ and the angle of $A$ at the vertex $V_{i}$ is $\delta_{i}$, then

$$
p_{i} \alpha+q_{i} \beta+r_{i} \gamma=\delta_{i}
$$

If $i>n$, then we have either

$$
p_{i} \alpha+q_{i} \beta+r_{i} \gamma=2 \pi
$$

or

$$
p_{i} \alpha+q_{i} \beta+r_{i} \gamma=\pi
$$

Namely, (2) holds if $V_{i}$ is in the interior of $A$ and whenever $V_{i}$ is on the boundary of a triangle $\Delta_{j}$, then necessarily $V_{i}$ is a vertex of $\Delta_{j}$. In the other cases (3) will hold. It is clear that the coefficients $p_{i}, q_{i}, r_{i}$ must satisfy

$$
\sum_{i=1}^{m} p_{i}=\sum_{i=1}^{m} q_{i}=\sum_{i=1}^{m} r_{i}=N .
$$


The tiling $T$ will be called regular, if one of the following statements is true:

$$
\begin{aligned}
& p_{i}=q_{i} \text { for every } i=1, \ldots, m ; \\
& p_{i}=r_{i} \text { for every } i=1, \ldots, m ; \\
& q_{i}=r_{i} \text { for every } i=1, \ldots, m .
\end{aligned}
$$

Otherwise the tiling is called irregular. We shall denote by $s_{1}(A)\left(s_{2}(A)\right)$ the number of distinct nonsimilar triangles $\Delta$ such that $A$ has a regular (irregular) tiling with triangles similar to $\Delta$. Our main results are the following:

Theorem 3. Let $A$ be a polygon such that $s_{1}(A)>0$. Then there is an angle, $\delta$, of $A$ such that $A$ can be decomposed into finitely many symmetric trapezoids with angles $\delta$ and $\pi-\delta$.

Theorem 4. For every polygon $A$ we have $s_{2}(A) \leq c n^{6}$, where $n$ is the number of vertices of $A$, and $c$ is an absolute constant.

Since $s(A) \leq s_{1}(A)+s_{2}(A)$, the following result is an immediate consequence of Theorems 3 and 4:

Corollary 5. For every polygon $A$, if $s(A)=\infty$, then there is an angle, $\delta$, of $A$ such that $A$ can be decomposed into finitely many symmetric trapezoids with angles $\delta$ and $\pi-\delta$. Moreover, the same conclusion holds if $s(A)>c n^{6}$, where $n$ is the number of vertices of $A$, and $c$ is an absolute constant.

First we prove Theorem 3. In the next three lemmas $\gamma$ and $\delta$ are fixed positive numbers such that $\gamma \neq \pi / 2$ and $\gamma+\delta=\pi$. For the sake of brevity we shall use the term "trapezoid" to indicate symmetric trapezoids with angles $\gamma$ and $\delta$. By the "legs" of a trapezoid $T$ we mean the nonparallel sides of $T$.

Lemma 6. Every parallelogram with angles $\gamma$ and $\delta$ can be decomposed into at most four trapezoids.

Proof. If the longer side of the parallelogram $P$ is at least twice the other side, then a suitable line going through the center of $P$ will decompose $P$ into two congruent trapezoids. Otherwise we dissect $P$ into two thin congruent parallelograms, and then we decompose each of these parallelograms into two trapezoids.

Lemma 7. Let A be a polygon with angles $\delta_{1}, \ldots, \delta_{2 n}$ such that $\delta_{i} \in\{\gamma, \gamma+\pi, \delta, \delta+\pi\}$ for every $i=1, \ldots, 2 n$, and $\left|\left\{i: \delta_{i} \in\{\gamma, \gamma+\pi\}\right\}\right|=n$. Then $A$ can be decomposed into finitely many trapezoids.

Proof. Let $V_{1}, \ldots, V_{2 n}, V_{2 n+1}=V_{1}$ denote the vertices of $A$, and let $i$ be an index such that $\delta_{i}=\gamma$ or $\gamma+\pi$ and $\delta_{i+1}=\delta$ or $\delta+\pi$. Then the sides $V_{i-1} V_{i}$ and $V_{i+1} V_{i+2}$ are parallel to each other. Let $V_{i}=P_{0}, P_{1}, \ldots, P_{m}=V_{i+1}$ be a division of the segment 
$V_{i} V_{i+1}$ into $m$ equal parts. We construct congruent nonoverlapping trapezoids $T_{1}, \ldots, T_{m}$ such that:

(i) $P_{j-1} P_{j}$ is one of the legs of $T_{j}$ for every $j=1, \ldots, m$;

(ii) the trapezoids $T_{1}, \ldots, T_{m}$ lie in the "inner side" of the segment $V_{i} V_{i+1}$; and

(iii) the bases of $T_{1}, \ldots, T_{m}$ are parallel to the sides $V_{i-1} V_{i}$ and $V_{i+1} V_{i+2}$.

If we choose $m$ large enough, and the diameter of $T_{j}$ small enough, then the trapezoids $T_{j}$ will be covered by $A$. We fix the trapezoids $T_{1}, \ldots, T_{m}$ with these properties. If we delete $T_{1}, \ldots, T_{m}$ from $A$, then we obtain a polygon $A^{\prime} \subset A$. The boundary of $A^{\prime}$ is obtained from that of $A$ by replacing the segments $V_{i-1} V_{i}, V_{i} V_{i+1}$, and $V_{i+1} V_{i+2}$ by the polygonial line $V_{i-1} U_{1} U_{2} V_{i+2}$, where $U_{1}$ is a point of the ray $\vec{V}_{i-1} V_{i}, U_{2}$ is a point of the ray $\overrightarrow{V_{i+2} V_{i+1}}$, and $U_{1} U_{2}$ is a zig-zag line covered by the boundaries of $T_{1}, \ldots, T_{m}$.

This operation of constructing and deleting the trapezoids $T_{1}, \ldots, T_{m}$ will be called a reduction of $A$ along the side $V_{i} V_{i+1}$. The resulting polygon will be called a reduced polygon of $A$. We define a similar reduction process if $\delta_{i}=\delta$ or $\delta+\pi$ and $\delta_{i+1}=\gamma$ or $\gamma+\pi$. Let

$$
\varepsilon_{i}=\left\{\begin{array}{ll}
0 & \text { if } \delta_{i}=\gamma \text { or } \gamma+\pi \\
1 & \text { if } \delta_{i}=\delta \text { or } \delta+\pi
\end{array} \quad(i=1, \ldots, 2 n) .\right.
$$

We may assume, by shifting the indices of the vertices if necessary, that $\varepsilon_{1}=0$ and $\varepsilon_{2 n}=1$. The sequence $\varepsilon_{1}, \ldots, \varepsilon_{2 n}$ is called the $0-1$ sequence corresponding to $A$. Suppose that $\varepsilon_{i}=0$ and $\varepsilon_{i+1}=1$. It is easy to check that if we apply a reduction along the side $V_{i} V_{i+1}$, then the $0-1$ sequence corresponding to the reduced polygon $A^{\prime}$ will be of the form $\varepsilon_{1}, \ldots, \varepsilon_{i-1} 1,0,1,0, \ldots, 1,0, \varepsilon_{i+2}, \ldots, \varepsilon_{2 n}$. Similarly, if $\varepsilon_{i}=1$ and $\varepsilon_{i+1}=0$, then the $0-1$ sequence corresponding to the reduced polygon $A^{\prime}$ will be of the form $\varepsilon_{1}, \ldots, \varepsilon_{i-1} 0,1,0,1, \ldots, 0,1, \varepsilon_{i+2}, \ldots, \varepsilon_{2 n}$.

Since $\varepsilon_{1}=0$ and $\varepsilon_{2 n}=1$, there are indices $1=i_{1}<i_{2}<\cdots<i_{2 k}<2 n+1=i_{2 k+1}$ such that $\varepsilon_{i}=0$ for every $i_{2 j-1} \leq i \leq i_{2 j}-1(j=1, \ldots, k)$ and $\varepsilon_{i}=1$ for every $i_{2 j} \leq i \leq i_{2 j+1}-1(j=1, \ldots, k)$. We shall prove the lemma by induction on the value of $n-k$.

If $n-k=0$, then $\varepsilon_{i}=0$ or 1 according to whether $i$ is odd or even; that is, $\delta_{i}=\gamma$ or $\gamma+\pi$ if $i$ is odd and $\delta_{i}=\delta$ or $\delta+\pi$ if $i$ is even. Since $\gamma+\delta=\pi$, this implies that the sides of $A$ represent only two directions enclosing the angle $\gamma$. From this it follows that $A$ can be decomposed into finitely many parallelograms of angles $\gamma$ and $\delta$ and then the statement follows from Lemma 6.

Now suppose that $n-k>0$. We shall prove that, applying finitely many reductions along suitably chosen sides, the value $n-k$ can be reduced. Clearly, this will finish the proof.

The condition $k<n$ implies that the sequence $\varepsilon_{1}, \ldots, \varepsilon_{2 n}$ contains two consequtive 0 's as well as two consecutive 1's. Let the indices $i$ and $j$ be such that $\varepsilon_{i}=\varepsilon_{i+1}=0$, $\varepsilon_{j}=\varepsilon_{j+1}=1$, and $|i-j|$ is minimal among these pairs $(i, j)$. We may assume that $i<j$, since the case $i>j$ can be treated similarly. If $j=i+2$, then $\left(\varepsilon_{i}, \varepsilon_{i+1}, \varepsilon_{i+2}, \varepsilon_{i+3}\right)=$ $(0,0,1,1)$. If we apply a reduction along the side $V_{i+1}, V_{i+2}$, then the $0-1$ sequence corresponding to the reduced polygon will be of the form

$$
\varepsilon_{1}, \ldots, \varepsilon_{i-1}, 0,1,0,1,0, \ldots, 1,0,1, \varepsilon_{i+4}, \ldots, \varepsilon_{2 n} .
$$


It is easy to check that in this way we have reduced the value of $n-k$ by 1 . Next suppose $j>i+2$. Then, by the minimality of $|i-j|$, the sequence $\varepsilon_{i}, \varepsilon_{i+1}, \ldots, \varepsilon_{j}, \varepsilon_{j+1}$ looks like $0,0,1,0,1,0, \ldots, 1,0,1,1$. If we apply a reduction along the side $V_{j-1}, V_{j}$, then the $0-1$ sequence corresponding to the reduced polygon will be of the form

$$
\varepsilon_{1}, \ldots, \varepsilon_{i-1}, 0,0,1,0,1,0, \ldots, 1,1,0,1,0, \ldots, 1,0,1, \varepsilon_{j+2}, \ldots, \varepsilon_{2 n} \text {. }
$$

This reduction does not change the value of $n-k$. However, in the new sequence we have $\varepsilon_{j-2}=\varepsilon_{j-1}=1$, and thus we reduced the value of $|i-j|$. (That is, in the new sequence there is a pair of consecutive 0's and another pair of consecutive 1's closer to each other than in the sequence corresponding to $A$.) Iterating this process we arrive at a polygon with a $0-1$ sequence containing the configuration $0,0,1,1$. As we saw above, one more reduction produces a polygon with a smaller value of $n-k$.

Lemma 8. Let $A$ be a polygon with angles $\delta_{i}=p_{i} \delta+r_{i} \gamma(i=1, \ldots, k)$, where $p_{i}$ and $r_{i}$ are nonnegative integers such that $\sum_{i=1}^{k} p_{i}=\sum_{i=1}^{k} r_{i}$. Then A can be decomposed into finitely many trapezoids.

Proof. The angle $\delta_{i}$ will be called desirable, if the pair $\left(p_{i}, r_{i}\right)$ equals one of $(1,0),(0,1)$, $(1,2),(2,1)$. The other angles of $A$ will be called undesirable. We shall prove the lemma by induction on the number $N=\sum^{\prime}\left(p_{i}+r_{i}\right)$, where the summation is taken over those indices $i$ for which the angle $\delta_{i}$ is undesirable.

If $N=0$; that is, if each angle of $A$ is desirable, then, by $\sum_{i=1}^{k} p_{i}=\sum_{i=1}^{k} r_{i}$, the polygon $A$ satisfies the conditions of the previous lemma with $n=k / 2$. Therefore, in this case the statement of the lemma is true. We shall prove that if $N>0$ then, deleting a suitable trapezoid from $A$, the value of $N$ can be reduced, which will finish the proof.

Let $\delta_{i}=p_{i} \delta+r_{i} \gamma$ be an undesirable angle. By symmetry, we may assume that $p_{i} \geq r_{i}$. Since $\delta_{i} \neq \pi, 2 \pi$, this implies $p_{i}>r_{i}$. Thus either $p_{i}=2$ and $r_{i}=0$, or $p_{i} \geq 3$. Let $T \subset A$ be a small trapezoid such that $V_{i}$ is a vertex of $T$, one of the bases of $T$ lies on the side $V_{i-1} V_{i}$, and the angle of $T$ at the vertex $V_{i}$ is $\delta$. If we delete $T$ from $A$, then we obtain a polygon $A^{\prime}$. The angle of $A^{\prime}$ at $V_{i}$ equals $\left(p_{i}-1\right) \delta+r_{i} \gamma$. There are also three new vertices of $A^{\prime}$ with desirable angles; two of them equal $\delta+\pi=2 \delta+\gamma$, and the third is $\gamma$. This implies that the condition $\sum_{i=1}^{k} p_{i}=\sum_{i=1}^{k} r_{i}$ remains valid in $A^{\prime}$, while the value of $N$ becomes strictly smaller.

Proof of Theorem 3. The condition $s_{1}(A)>0$ means that there exists a regular tiling of $A$ with similar triangles. Let (1), (2), (3) be the equations corresponding to such a tiling. By permuting the angles $\alpha, \beta, \gamma$ if necessary, we may assume that $p_{i}=q_{i}$ for every $i=1, \ldots, m$. Let $\delta=\pi-\gamma$, then the left-hand side of each of the equations (1), (2), (3) can be written as $p_{i} \delta+r_{i} \gamma$.

Suppose first $\gamma=\pi / 2$. Then $\delta=\pi / 2$ and thus every angle of $A$ is either $\pi / 2$ or $3 \pi / 2$. In this case $A$ can be decomposed into rectangles, which proves the statement.

Next suppose $\gamma=\pi / 3$ or $2 \pi / 3$. Then $\delta=\pi / 3$ or $2 \pi / 3$, and thus every angle of $A$ is a multiple of $\pi / 3$. Then the elongations of the sides of $A$ dissect $A$ into nonoverlapping convex polygons with angles $\pi / 3$ and $2 \pi / 3$. Therefore, in this case, it is enough to show that every convex polygon $B$ with angles $\pi / 3$ and $2 \pi / 3$ can be decomposed into 
symmetric trapezoids of angles $\pi / 3$ and $2 \pi / 3$. We already proved this if $B$ is a triangle. If $B$ is a quadrilateral, then $B$ is either a symmetric trapezoid or a parallelogram. By Lemma 6 , in the latter case, $B$ can be decomposed into at most four symmetric trapezoids of angles $\pi / 3$ and $2 \pi / 3$. If $B$ is a pentagon, then one of its angles is $\pi / 3$ and the others are $2 \pi / 3$. It is easy to check by inspection that $B$ can be decomposed into at most three symmetric trapezoids of angles $\pi / 3$ and $2 \pi / 3$. Finally, if $B$ is a hexagon, then each angle of $B$ is $2 \pi / 3$, and we can decompose $B$ into at most four symmetric trapezoids of angles $\pi / 3$ and $2 \pi / 3$.

Therefore we may assume that $\gamma \neq \pi / 2, \pi / 3,2 \pi / 3$. Let $P=\sum_{i=1}^{n} p_{i}$ and $R=$ $\sum_{i=1}^{n} r_{i}$. If $P=R$ then, by Lemma $8, A$ can be decomposed into nonoverlapping trapezoids. The cases $P>R$ and $P<R$ can be treated similarly to each other, by exchanging the roles of $\gamma$ and $\delta$. Therefore we shall suppose $P>R$.

For every $i>n$ we have $p_{i} \delta+r_{i} \gamma=v_{i} \pi$, where $v_{i}=1$ or 2 . Let $k_{i}=r_{i}-p_{i}$ and $w_{i}=v_{i}-p_{i}$, then $k_{i} \gamma=w_{i} \pi(i>n)$. Since $P+\sum_{i=n+1}^{m} p_{i}=R+\sum_{i=n+1}^{m} r_{i}$ by (4), thus

$$
P-R=\sum_{i=n+1}^{m} k_{i},
$$

and hence $k_{j}>0$ for at least one $j>n$. Then necessarily $w_{j}=v_{j}-p_{j}>0$, and $w_{j}=1$ or 2 . Since $0<\gamma<\pi$ and $\gamma \neq \pi / 2, \pi / 3,2 \pi / 3$, this implies $k_{j} \geq 4$ and $\gamma<\pi / 2$. We claim that $k_{i} \geq 0$ for every $i>n$. Indeed, suppose $k_{i}<0$. Then $p_{i}>r_{i}$ and $\left(p_{i}-r_{i}\right) \delta=z_{i} \pi$, where $z_{i}=v_{i}-r_{i}$. Then $z_{i}>0, z_{i}=1$ or 2 . Since $\delta>\pi / 2$, this would imply $p_{i}-r_{i}=3$ and $z_{i}=2$, that is, $\delta=2 \pi / 3, \gamma=\pi / 3$, which is impossible. Therefore $k_{i} \geq 0$ and $w_{i} \in\{0,1,2\}$ for every $i>n$. We shall distinguish between the following two cases.

Case I: There is a $j>n$ with $w_{j}=1$.

Then fixing such a $j$ and putting $k=k_{j}$ we obtain $k \gamma=\pi$, and $k_{i}=w_{i} k$ for every $i>n$. This implies, by (5), that $P-R$ is an integer multiple of $k$. Let $P-R=s k$. We show first that either $\delta$ or $\gamma$ equals one of the angles of $A$. We have

$$
(n-2) \pi=P \delta+R \gamma=(R+s k)(\pi-\gamma)+R \gamma=(R+s k) \pi-s \pi .
$$

This implies $R=n-2-s k+s, P=n-2+s$, and $P+R=2 n-4-s k+2 s<2 n$, since $k \geq 4$. Therefore $p_{i}+r_{i}=1$ holds for at least one index $i$, and thus $\delta_{i}=p_{i} \delta+r_{i} \gamma$ equals either $\delta$ or $\gamma$.

We shall prove that, deleting from $A$ a suitable system of trapezoids, we can reduce the value of $P-R$ by $k$. Then, iterating this process we can produce a polygon with $P-R=0$ which, according to Lemma 8 , can be decomposed into nonoverlapping trapezoids.

Let $P_{1}$ and $P_{2}$ be inner points of any of the sides of $A$. Let $T_{1}$ be a trapezoid with vertices $P_{1}, P_{2}, P_{3}, P_{4}$ listed counterclockwise, such that $P_{1} P_{2}$ is the longer base of $T_{1}$ and the angle of $T_{1}$ at $P_{1}$ equals $\gamma$. If we rotate $T_{1}$ about the point $P_{1}$ by the angle $\gamma$ and apply a suitable homothetic transformation, we obtain a trapezoid $T_{2}$ such that the longer base of $T_{2}$ is $P_{1} P_{4}$. Rotating $T_{2}$ about $P_{1}$ by $\gamma$ and using the same homothetic transformation, we obtain a trapezoid $T_{3}$. Continuing this process we obtain the nonoverlapping trapezoids 
$T_{1}, \ldots, T_{k}$ such that the longer base of $T_{i}$ coincides with one of the legs of $T_{i-1}$ for every $i=2, \ldots, k$. Since $k \gamma=\pi$, the union of these trapezoids is a convex polygon $C$ with $k+2$ vertices. The angle of $C$ at the vertex $P_{2}$ equals $\gamma$, and the other angles of $C$ equal $\delta$.

With a suitable choice of the points $P_{1}$ and $P_{2}$, the polygon $C$ will be covered by $A$. Then, deleting $C$ from $A$ we obtain a polygon $A^{\prime}$ with $k+2$ new angles; one of them is $\delta$, another equals $\gamma$, and the others are $2 \pi-\delta=\delta+2 \gamma$. Putting $P^{\prime}=P+1+k$ and $R^{\prime}=R+1+2 k$, we find $P^{\prime}-R^{\prime}=P-R-k$. In this way we reduced the value of $P-R$ by $k$ and, as we saw above, this proves the theorem.

Case II: $w_{i}=0$ or 2 for every $i>n$.

We fix $j>n$ with $w_{j}=2$ and put $k=k_{j}$; then $k \gamma=2 \pi$, and $k_{i}=0$ or $k$ for every $i>n$. This implies, by (5), that $P-R$ is an integer multiple of $k$. Then either $\delta$ or $\gamma$ equals one of the angles of $A$; this can be proved in the same way as in Case I.

We shall prove that deleting from $A$ a suitable system of trapezoids we can reduce the value of $P-R$ by $k$. This will finish the proof in the same way as in Case I.

Let $P_{1}$ and $P_{2}$ be inner points of any of the sides of $A$. Let $T_{1}$ be a trapezoid with vertices $P_{1}, P_{2}, P_{3}, P_{4}$, listed counterclockwise, such that $P_{1} P_{2}$ is the shorter base of $T_{1}$ and the angle of $T_{1}$ at $P_{3}$ equals $\gamma$. If we rotate $T_{1}$ about the point $P_{3}$ by the angle $\gamma$ and apply a suitable homothetic transformation, we obtain a trapezoid $T_{2}$ such that the longer base of $T_{2}$ is $P_{2} P_{3}$. Rotating $T_{2}$ about $P_{3}$ by $\gamma$ and using the same homothetic transformation, we obtain a trapezoid $T_{3}$. Continuing this process we obtain the nonoverlapping trapezoids $T_{1}, \ldots, T_{k}$ such that the longer base of $T_{i}$ coincides with one of the legs of $T_{i-1}$ for every $i=2, \ldots, k$. Since $k \gamma=2 \pi$, the union of these trapezoids is a nonconvex polygon $D$ with $k+2$ vertices. The angle of $D$ at the vertex $P_{4}$ equals $\gamma$, another angle of $D$ equals $\delta+\pi$, and the other angles of $D$ equal $\delta$.

With a suitable choice of the points $P_{1}$ and $P_{2}$ the polygon $D$ will be covered by $A$. Then, deleting $D$ from $A$ we obtain a polygon $A^{\prime}$ with $k+2$ new angles; three of them equal $\gamma$, another equals $2 \pi-\gamma=2 \delta+\gamma$, and the others are $2 \pi-\delta=\delta+2 \gamma$. Putting $P^{\prime}=P+2+(k-2)$ and $R^{\prime}=R+3+1+(k-2) \cdot 2$, we find $P^{\prime}-R^{\prime}=P-R-k$, which completes the proof.

\section{Proof of Theorem 4}

Let $\delta_{1}, \ldots, \delta_{n}$ denote the angles of $A$, let $\alpha, \beta, \gamma$ be the angles of $\Delta$, and suppose that $A$ has an irregular tiling with similar copies of $\Delta$. We put $a_{i}=\delta_{i} / \pi(i=1, \ldots, n), x=$ $\alpha / \pi, y=\beta / \pi, z=\gamma / \pi$; then we have $0<a_{i}<2, a_{i} \neq 1(i=1, \ldots, n)$, $\sum_{i=1}^{n} a_{i}=n-2, x, y, z>0$, and $x+y+z=1$. Theorem 4 is an immediate consequence of the following lemma:

Lemma 9. Let $a_{1}, \ldots, a_{n} \in(0,2), a_{i} \neq 1(n=1, \ldots, n)$ be given such that $\sum_{i=1}^{n} a_{i}<n$. Then there are at most $c n^{6}$ triples $(x, y, z)$ with the following properties: $x, y, z>0, x+y+z=1$, and there are nonnegative integers $p_{i}, q_{i}, r_{i}(i=$ $1, \ldots, m ; m \geq n)$ such that:

(i) $p_{i} x+q_{i} y+r_{i} z=a_{i}(i=1, \ldots, n)$; 
(ii) $p_{i} x+q_{i} y+r_{i} z=b_{i} \in\{1,2\}(i=n+1, \ldots, m)$;

(iii) $\sum_{i=1}^{m} p_{i}=\sum_{i=1}^{m} q_{i}=\sum_{i=1}^{m} r_{i}$; and

(iv) there are indices $i, j, k$ such that $p_{i} \neq q_{i}, p_{j} \neq r_{j}, q_{k} \neq r_{k}$.

Proof. We shall prove slightly more: we show that if we replace (i) by the weaker condition

(i') $p_{i} x+q_{i} y+r_{i} z=a_{i}$ or $p_{i} x+q_{i} y+r_{i} z=a_{i}-1$ for every $i=1, \ldots, n$,

then the statement of the lemma is still true. Let $(x, y, z)$ be a given triple with the required properties. We fix the numbers $m, p_{i}, q_{i}, r_{i}(i=1, \ldots, m)$ and $b_{i}(i=$ $n+1, \ldots, m)$ satisfying the conditions (i'), (ii), (iii), (iv) and such that the value of $N=\sum_{i=1}^{m} p_{i}=\sum_{i=1}^{m} q_{i}=\sum_{i=1}^{m} r_{i}$ is minimal. Then $\min \left(p_{i}, q_{i}, r_{i}\right)=0$ for every $i$. Indeed, if $\min \left(p_{i}, q_{i}, r_{i}\right)>0$ and $i \leq n$, then necessarily $p_{i} x+q_{i} y+r_{i} z=a_{i}>1$ (note that $a_{i} \neq 1$ by assumption), and then we could replace the equation $p_{i} x+q_{i} y+r_{i} z=a_{i}$ by $\left(p_{i}-1\right) x+\left(q_{i}-1\right) y+\left(r_{i}-1\right) z=a_{i}-1$. This replacement does not violate the conditions (i'), (ii), (iii), (iv), but decreases the value of $N$ which is impossible. Next, if $\min \left(p_{i}, q_{i}, r_{i}\right)>0$ and $i>n$, then there are two cases. If $b_{i}=1$, then necessarily $p_{i}=q_{i}=r_{i}=1$ and we could delete the equation $p_{i} x+q_{i} y+r_{i} z=b_{i}$ from the system. If $b_{i}=2$, then we replace $p_{i} x+q_{i} y+r_{i} z=2$ by $\left(p_{i}-1\right) x+\left(q_{i}-1\right) y+\left(r_{i}-1\right) z=1$. Both changes would reduce the value of $N$, a contradiction.

We shall denote by $E_{i}$ the equation $p_{i} x+q_{i} y+r_{i} z=c_{i}$, where $c_{i}=a_{i}$ or $a_{i}-1$ if $i \leq n$ and $c_{i}=b_{i}$ if $i>n$.

\section{Lemma 10. The determinant}

$$
D_{i j}=\left|\begin{array}{ccc}
1 & 1 & 1 \\
p_{i} & q_{i} & r_{i} \\
p_{j} & q_{j} & r_{j}
\end{array}\right|
$$

is nonzero for at least one pair of indices $i, j \leq m$.

Proof. Suppose $D_{i j}=0$ for every $i, j \leq m$. Then for every $i$, exactly one of the numbers $p_{i}, q_{i}, r_{i}$ is zero. Indeed, if, say, $q_{i}=r_{i}=0$ for some $i \leq m$, then $D_{i j}=0$ gives $q_{j}=r_{j}$ for every $j$, which contradicts condition (iv).

Let $i$ be fixed with $a_{i}<1$ (such an index exists by $\sum_{i=1}^{n} a_{i}<n$ ). We may suppose, by symmetry, that $r_{i}=0$ and $p_{i}, q_{i}>0$. Let $j$ be an index with $r_{j}>0$. Then exactly one of $p_{j}$ and $q_{j}$ is zero; we shall assume that $p_{j}>0$ and $q_{j}=0$. The condition $D_{i j}=0$ implies that the equation $x+y+z=1$ is a linear combination of $E_{i}$ and $E_{j}$. Then we have

$$
1=\frac{p_{i}}{q_{i}}+\frac{p_{j}}{r_{j}}=\frac{a_{i}}{q_{i}}+\frac{c_{j}}{r_{j}}<\frac{1}{q_{i}}+\frac{2}{r_{j}},
$$

as $a_{i}<1$ and $c_{j} \leq 2$. Since $p_{i}, p_{j}, q_{i}, r_{j}>0$, this implies $p_{i} / q_{i}<1$ and $p_{j} / r_{j}<1$. Therefore $q_{i}, r_{j} \geq 2$,

$$
1<\frac{1}{q_{i}}+\frac{2}{r_{j}} \leq \frac{1}{2}+\frac{2}{r_{j}}
$$


and $r_{j} \leq 3$. Now $r_{j}=3$ is impossible by (7), and thus we have $r_{j}=2, p_{j}=1$, and $p_{i} / \bar{q}_{i}=\frac{1}{2}$. Since $j$ was any index with $r_{j}>0$, this shows that $r_{j}>0$ implies $p_{j}=1, q_{j}=0, r_{j}=2$. On the other hand, if $r_{j}=0$, then $D_{i j}=0$ gives $p_{j} / q_{j}=1 / 2$.

Let $R=\left\{j: r_{j}>0\right\}$, then $N=\sum_{j \in R} r_{j}=2|R|$ and thus $|R|=N / 2$. Since $r_{i}=0$, we have

$$
p_{i}(x+2 y)=p_{i} x+q_{i} y=a_{i}<1,
$$

and thus $x+2 y<1=x+y+z, y<z, x+2 z>1$. Consequently, if $j \in R$ then $c_{j}=x+2 z \in(1,2)$ and hence $j \leq n$ (note that $b_{j} \in\{1,2\}$ ). That is, $R \subset\{1, \ldots, n\}$. Let $P=\sum_{j \in\{1, \ldots, n\} \backslash R} p_{j}$. Then we have

$$
|P| \geq|\{1, \ldots, n\} \backslash R|=n-|R|=n-\frac{N}{2} \quad \text { and } \quad P=\frac{1}{2} \cdot \sum_{j \in\{1, \ldots, n\} \backslash R} q_{j} \leq \frac{N}{2} .
$$

On the other hand, putting $u=x+2 y$, we obtain, by $u<1$,

$$
\begin{aligned}
n>\sum_{j=1}^{n} a_{j} & =\sum_{j \in R}(x+2 z)+\sum_{j \in\{1, \ldots, n\} \backslash R}\left(p_{j}+2 p_{j} y\right)=|R|(x+2 z)+P(x+2 y) \\
& =\frac{N}{2}(2-u)+P u=N-\left(\frac{N}{2}-P\right) u \geq N-\left(\frac{N}{2}-P\right) \\
& =P+\frac{N}{2} \geq n,
\end{aligned}
$$

a contradiction.

In order to prove Lemma 9, we shall distinguish between the following two cases:

Case I: $N \leq 120$.

In this case $p_{i}, q_{i}, r_{i} \leq 120 n$ for every $i \leq m$. Let $i, j$ be such that $D_{i j} \neq 0$. Then the system of equations $x+y+z=1, E_{i}$ and $E_{j}$ determine $x, y, z$; namely $x=$ $D_{x} / D_{i j}, y=D_{y} / D_{i j}$, and $z=D_{z} / D_{i j}$, where $D_{x}, D_{y}, D_{z}$ are determinants involving the numbers $1, c_{i}, c_{j}, p_{i}, q_{i}, r_{i}, p_{j}, q_{j}, r_{j}$. Now $c_{i}$ and $c_{j}$ can take at most $2 n+2$ values (namely $a_{k}, a_{k}-1(k=1, \ldots, n)$ and 1,2$)$. On the other hand, each of the numbers $p_{i}, q_{i}, r_{i}, p_{j}, q_{j}, r_{j}$ can take at most $120 n+1$ possible values, and at least two of them are zero. This implies that in this case the number of possible triples $(x, y, z)$ is at most

$$
(2 n+2)^{2} \cdot\left(\begin{array}{l}
6 \\
2
\end{array}\right) \cdot(120 n+1)^{4} \leq c \cdot n^{6} .
$$

Case II: $N>120 n$.

We prove that in this case $x, y, z$ are rational numbers with denominator not exceeding 2100. Clearly, this will finish the proof. Recall that the determinant $D_{i j}$ was defined in (6).

Lemma 11. Suppose that $i, j>n, \min \left(\max \left(p_{i}, p_{j}\right), \max \left(q_{i}, q_{j}\right), \max \left(r_{i}, r_{j}\right)\right)>0$, and $\max \left(\min \left(p_{i}, p_{j}\right), \min \left(q_{i}, q_{j}\right), \min \left(r_{i}, r_{j}\right)\right)>0$. If $D_{i j}=0$, then $p_{i}+p_{j}=q_{i}+q_{j}=$ $r_{i}+r_{j}$. 
Proof. The condition $\min \max >0, \max \min >0$ implies that, after a suitable permutation of $i, j$ and $x, y, z$ we have $p_{i}>0, q_{i}=0, r_{i}>0$ and $p_{j}=0, q_{j}>0, r_{j}>0$. If $D_{i j}=0$, then the equation $x+y+z=1$ is a linear combination of $E_{i}$ and $E_{j}$. This implies that

$$
1=\frac{r_{i}}{p_{i}}+\frac{r_{j}}{q_{j}}=\frac{b_{i}}{p_{i}}+\frac{b_{j}}{q_{j}} .
$$

Suppose $p_{i} \neq q_{j}$. Then we may assume $p_{i}<q_{j}$, as the roles of $x$ and $y$ are symmetric. Since $r_{i}, r_{j}$ and $p_{i}<q_{j}$ are positive integers and $b_{i}, b_{j} \in\{1,2\},(8)$ implies that only the following cases are possible:

$$
\begin{aligned}
& E_{i}: 2 x+z=1, E_{j}: 4 y+2 z=2 \\
& E_{i}: 3 x+z=2, E_{j}: 6 y+4 z=2 \\
& E_{i}: 3 x+2 z=2, E_{j}: 6 y+2 z=2 .
\end{aligned}
$$

Then we can replace $E_{i}$ and $E_{j}$ by a single equation $R$ in such a way that the conditions (i'), (ii), (iii), (iv) remain valid, but the value of $N$ is reduced. Indeed, let $R$ be, in the respective cases, $2 y+z=1,3 y+2 z=1$, or $3 y+z=1$. Since $N$ was minimal, this is absurd, and thus $p_{i} \neq q_{j}$ is impossible. Thus we have $p_{i}=q_{j}$. By (8) this implies $p_{i}=q_{j}=r_{i}+r_{j}$, which was to be proved.

Lemma 12. Suppose $N>8 n+16$. If there are indices $i, j>n$ such that $\min \left(\max \left(p_{i}, p_{j}\right), \max \left(q_{i}, q_{j}\right), \max \left(r_{i}, r_{j}\right)\right)>0$, then $x, y, z$ are rational numbers with denominator not greater than $\max \left(\left|D_{i j}\right|, 50\right)$.

Proof. Suppose first that $\max \left(\min \left(p_{i}, p_{j}\right), \min \left(q_{i}, q_{j}\right), \min \left(r_{i}, r_{j}\right)\right)=0$. Then, after a suitable permutation of $i, j$ and $x, y, z$ we have $p_{i}>0, q_{i}=r_{i}=0$, and $p_{j}=$ $0, q_{j}>0, r_{j}>0$. If $D_{i j} \neq 0$ then, by Cramer's rule, $x, y, z$ are rational numbers with denominator $\left|D_{i j}\right|$. Therefore we may assume $D_{i j}=0$; that is, $q_{j}=r_{j}$. Then the equations $E_{i}$ and $E_{j}$ look like $p_{i} x=b_{i}$ and $q_{j}(y+z)=b_{j}$, where $b_{i}, b_{j} \in\{1,2\}$.

If $p_{i}=q_{j}$, then by adding $E_{i}$ and $E_{j}$ we obtain $p_{i}(x+y+z)=b_{i}+b_{j}$ which implies $p_{i} \leq 4$ and $x=b_{i} / p_{i} \geq \frac{1}{4}$. If we delete $E_{i}$ and $E_{j}$ from the system, then conditions (i'), (ii), and (iii) remain valid. Since $N$ was minimal, this implies that (iv) is violated. The existence of an index $k$ with $q_{k} \neq r_{k}$ remains true, since $q_{i}=r_{i}$ and $q_{j}=r_{j}$. Thus either $p_{k}=q_{k}$ for every $k \neq i, j$, or $p_{k}=r_{k}$ for every $k \neq i, j$. By symmetry, we may suppose the former.

Since $x \geq \frac{1}{4}$, we have $p_{k} \leq 8$ for every $k$ and thus $\sum_{k>n} p_{k} \geq N-8 n>16$. This implies that there is a $k>n$ with $k \neq i, j$ and $p_{k}>0$. Then $p_{k}=q_{k}$ and $p_{k}(x+y) \in\{1,2\}$. Thus $x+y$ is rational with denominator not greater than 8 . Then each of $x, y, z$ is rational with denominator $\leq 32$, and the statement of the lemma is true.

Therefore we may suppose $p_{i} \neq q_{j}$. Since either $x \geq \frac{1}{2}$ or $y+z>\frac{1}{2}$, we have either $p_{i} \leq 4$ or $q_{j} \leq 3$. Then we find that the possible cases are the following:

$$
\begin{aligned}
& E_{i}: 2 x=1, E_{j}: 4(y+z)=2 ; \\
& E_{i}: 4 x=2, E_{j}: 2(y+z)=1 ;
\end{aligned}
$$




$$
\begin{aligned}
& E_{i}: 3 x=2, E_{j}: 6(y+z)=2 \\
& E_{i}: 6 x=2, E_{j}: 3(y+z)=2
\end{aligned}
$$

In these cases we replace these pairs of equations, in turn, by $2 y+2 z=1,2 x=$ $1,3(y+z)=1$, and $3 x=1$. Using the previous argument we can see that in this case $x, y, z$ are again rationals with small denominator. This finishes the proof supposing $\max \left(\min \left(p_{i}, p_{j}\right), \min \left(q_{i}, q_{j}\right), \min \left(r_{i}, r_{j}\right)\right)=0$.

If $\max \left(\min \left(p_{i}, p_{j}\right), \min \left(q_{i}, q_{j}\right), \min \left(r_{i}, r_{j}\right)\right)>0$ then, after a suitable arrangement we may suppose $p_{i}>0, q_{i}=0, r_{i}>0$ and $p_{j}=0, q_{j}>0, r_{j}>0$.

If $D_{i j} \neq 0$, then the equations $x+y+z=1, E_{i}, E_{j}$ determine $x, y, z$, and, by Cramer's rule, $x, y, z$ are rational numbers with denominator $D_{i j}$. Therefore, in this case the statement of the lemma is true. If $D_{i j}=0$ then, by Lemma 11, we have $p_{i}=q_{j}=r_{i}+r_{j}$. Deleting the equations $E_{i}, E_{j}$ from the system, conditions (i'), (ii), and (iii) remain valid. Since $N$ was minimal, this implies that (iv) does not remain true. That is, one of the equalities $p_{k}=q_{k}, p_{k}=r_{k}, q_{k}=r_{k}$ holds for every $k \neq i, j$.

Case I: $p_{k}=q_{k}$ for every $k \neq i, j$.

Suppose first $z \leq \frac{1}{3}$. Then $x+y \geq \frac{2}{3}$ and either $x \geq \frac{1}{3}$ or $y \geq \frac{1}{3}$. By symmetry we may suppose the former. Then $p_{k} \leq 6$ for every $k$ and, as we saw above, this implies the existence of a $k>n$ with $k \neq i, j$ and $p_{k}>0$. Then $p_{k}(x+y)=b_{k}$ and thus $x+y$ and $z$ are rationals with denominator $\leq 6$. Substituting this value of $z$ into $E_{i}$ and $E_{j}$ we obtain that $x$ and $y$ are rational numbers with denominator $\leq 36$.

Next suppose $z>\frac{1}{3}$. Then $r_{k} \leq 5$ for every $k$, and hence there is a $k>n, k \neq i, j$, such that $r_{k}>0$. Then $p_{k}=q_{k}=0, r_{k} z=b_{k}$, and $z$ is a rational with denominator $\leq 5$. Substituting into $E_{i}$ and $E_{j}$ we obtain that $x, y$ are rationals with denominator $\leq 5 p_{i}$. Since $p_{i}=r_{i}+r_{j} \leq 10$, these denominators are not greater than 50 .

Case II: $p_{k}=r_{k}$ for every $k \neq i, j$.

Suppose first $z \geq \frac{1}{3}$. Then $r_{k} \leq 6$ for every $k$, and thus there is a $k>n, k \neq i, j$ such that $r_{k}>0$. Then $p_{k}=r_{k}$ implies $q_{k}=0$, and thus $r_{k}(x+z)=b_{k}$. Therefore $x+z$ and $y$ are rationals with denominator $\leq 6$. Substituting into $E_{j}$ we find, as above, that $x, y, z$ are rationals with small denominator.

Next suppose $x \geq \frac{1}{3}$. Then $p_{k} \leq 6$ for every $k$, and thus there is a $k>n, k \neq i, j$ such that $p_{k}>0$. Then $p_{k}=r_{k}$ implies $q_{k}=0$, and we can argue as above.

Suppose finally $y \geq \frac{1}{3}$. Then $q_{k} \leq 6$ for every $k$, and thus there is a $k>n, k \neq i, j$ such that $q_{k}>0$. Then $p_{k}=r_{k}=0$ and $q_{k} y=b_{k}$. Thus $y$ is rational with denominator $\leq 6$. Substituting into $E_{j}$ we find that $z$ is also rational with small denominator.

Case III: $q_{k}=r_{k}$ for every $k \neq i, j$.

This is analogous to Case II.

The next lemma will complete the proof of Theorem 3.

Lemma 13. If $N>120 n$, then $x, y, z$ are rational numbers with denominator not greater than 2100 . 
Proof. In the sequel we shall assume that $z$ is the largest among $x, y, z$; then $z \geq \frac{1}{3}$. We shall also assume that $z>\frac{1}{3}$; that is, we exclude the case $z=\frac{1}{3}$. This is justified, since $z=\frac{1}{3}$ implies $x=y=z=\frac{1}{3}$. This assumption implies that $r_{i} \leq 5$ for every $i$.

Suppose first $z>\frac{2}{3}$. Let $R_{1}=\left\{i: i>n, p_{i}>0, r_{i}>0\right\}$ and $R_{2}=\{i: i>$ $\left.n, q_{i}>0, r_{i}>0\right\}$. Since $z>\frac{2}{3}$, there is no $i>n$ with $p_{i}=q_{i}=0$; that is, $R_{1} \cup R_{2}=\left\{i: i>n, r_{i}>0\right\}$. Also, $z>\frac{2}{3}$ implies that $r_{i} \leq 2$ for every $i$. Since $x+z=b_{i}, x+2 z=b_{i}, 2 x+2 z=b_{i}$ are impossible, we have $p_{i}>r_{i}$ for every $i \in R_{1}$. Then, by $r_{i} \leq 2$, we have $p_{i} \geq \frac{3}{2} r_{i}$ for $i \in R_{1}$. Therefore

$$
\sum_{i \in R_{1}} r_{i} \leq \frac{2}{3} \sum_{i \in R_{1}} p_{i} \leq \frac{2}{3} N
$$

and thus

$$
\sum_{i \in R_{2}} r_{i}=\sum_{i=n+1}^{m} r_{i}-\sum_{i \in R_{1}} r_{i} \geq(N-2 n)-\frac{2}{3} N>\frac{N}{4} .
$$

This implies $\left|R_{2}\right| \geq N / 8$. The same argument gives $\left|R_{1}\right| \geq N / 8$. Since

$$
\sum_{i \in R_{1}} p_{i} \leq N \quad \text { and } \quad \sum_{i \in R_{2}} q_{i} \leq N
$$

there must be indices $i \in R_{1}, j \in R_{2}$ such that $0<p_{i} \leq 8$ and $0<q_{j} \leq 8$. Therefore, by Lemma 12, $x, y, z$ are rationals with denominator $\leq \max \left(\left|D_{i j}\right|, 50\right)<100$.

Next suppose $z \leq \frac{2}{3}$. By symmetry we may assume that $x \leq y$. Then $x+y=1-z \geq \frac{1}{3}$ implies that $y \geq \frac{1}{6}$, and thus $q_{i} \leq 12$ for every $i$. Therefore $\sum_{j=n+1}^{m} q_{j} \geq N-12 n>N / 2$ and, consequently, there is a $j>n$ such that $0<q_{j} \leq 12$ and $p_{j} \leq 24$. Similarly, there is an $\ell>n$ such that $r_{\ell}>0$ and $p_{\ell} \leq 24$. Suppose first that there is an $i>n$ such that $0<p_{i} \leq 120$. If any of the equations $E_{i}, E_{j}, E_{\ell}$ contains two positive coefficients then, by Lemma 12, $x, y, z$ are rationals with denominator not exceeding

$$
\max \left(D_{i j}, D_{i \ell}, D_{j \ell}, 50\right) \leq 2100 .
$$

Otherwise we have $q_{j} y=b_{j}, p_{i} x=b_{i}, r_{\ell} z=b_{\ell}$, so that $x, y, z$ are rationals with denominator $\leq 120$.

Therefore we may assume that for every $i>n$, either $p_{i}=0$ or $p_{i}>120$ holds. Let

$$
P_{1}=\left\{i>n: p_{i}>0\right\} \quad \text { and } \quad P_{2}=\left\{i>n: p_{i}=0\right\},
$$

then $p_{i}>120$ for every $i \in P_{1}$ and hence $\left|P_{1}\right|<N / 120$. If there are indices $i, j \in P_{2}$ with $q_{i} / r_{i} \neq q_{j} / r_{j}$, then the equations $q_{i} y+r_{i} z=b_{i}, q_{j} y+r_{j} z=b_{j}$ imply that $y$ and $z$ (and then $x$ also) are rational numbers with denominator $\left|q_{i} r_{j}-r_{i} q_{j}\right|$. Since $q_{i}, q_{j}, r_{i}, r_{j} \leq 12$, this denominator is not greater than 144 .

Thus we may suppose that the ratio $\lambda=q_{i} / r_{i}$ is the same for every $i \in P_{2}$. Since $1>y+z>\frac{2}{3}, 2>2 y+2 z>\frac{4}{3}$, and $3 y+3 z>2, \lambda=1$ is impossible. Suppose $\lambda>1$. Then $q_{i}>r_{i}$ for every $i \in P_{2}$. Since $r_{i} \leq 5$, this implies $q_{i} \geq \frac{6}{5} r_{i}$ for every $i \in P_{2}$. Thus

$$
\sum_{i \in P_{2}} r_{i} \leq \frac{5}{6} \sum_{i \in P_{2}} q_{i} \leq \frac{5}{6} N
$$


On the other hand,

$$
\sum_{i \in P_{1}} r_{i} \leq 5 \cdot\left|P_{1}\right|<\frac{5 N}{120}
$$

and thus

$$
N=\sum_{i=1}^{m} r_{i}=\sum_{i=1}^{n} r_{i}+\sum_{i \in P_{2}} r_{i}+\sum_{i \in P_{1}} r_{i} \leq 5 n+\frac{5 N}{6}+\frac{N}{24} .
$$

This gives $N / 8 \leq 5 n$ and $N \leq 40 n$, contradicting the assumption.

If $\lambda<1$, then $q_{i}<r_{i} \leq 5$ for every $i \in P_{2}$ and hence $q_{i} \leq \frac{4}{5} r_{i}\left(i \in P_{2}\right)$. Therefore

$$
\sum_{i \in P_{2}} q_{i} \leq \frac{4}{5} \sum_{i \in P_{2}} r_{i} \leq \frac{4}{5} N
$$

On the other hand,

$$
\sum_{i \in P_{1}} q_{i} \leq 12 \cdot\left|P_{1}\right|<\frac{12 N}{120}=\frac{N}{10}
$$

and thus

$$
N=\sum_{i=1}^{m} q_{i}=\sum_{i=1}^{n} q_{i}+\sum_{i \in P_{2}} q_{i}+\sum_{i \in P_{1}} q_{i}<12 n+\frac{4 N}{5}+\frac{N}{10} .
$$

This gives $N / 10<12 n, N<120 n$, contradicting our assumption.

\section{References}

1. S. W. Golomb, Replicating figures in the plane, Math. Gaz., 48 (1964), 403-412.

2. M. Laczkovich, Tilings of polygons with similar triangles, Combinatorica, 10 (1990), 281-306.

3. M. Laczkovich, Tilings of triangles, Discrete Math., 140 (1995), 79-94.

4. M. Laczkovich and G. Szekeres, Tilings of the square with similar rectangles, Discrete Comput. Geom., 13 (1995), 569-572.

5. S. L. Snover, C. Waiveris, and J. K. Williams, Rep-tiling for triangles, Discrete Math., 91 (1991), 193-200.

6. A. Soifer, How Does One Cut a Triangle? Center for Excellence in Mathematical Education, Colorado Springs, 1990.

7. S. K. Stein and S. Szabó, Algebra and Tiling. The Carus Mathematical Monographs, No. 25, Mathematical Association of America, Washington, DC, 1994.

Received February 17, 1997, and in revised form June 16, 1997. 